\title{
Gradation theory of asphalt mixture aggregate based on fractal theory
}

\author{
Ruihua Yang ${ }^{1}$, Qiancheng Zhou ${ }^{2}$ \\ (1. Shanghai Urban Construction Vocational College; Shanghai 201999; China; 2. Shanghai \\ Technical Institute of Electronics \& Information; Shanghai 201411; China.)
}

Key words: road engineering, asphalt mixture, gradation theory, fractal

\begin{abstract}
This paper introduces the method to calculating fractal dimension of aggregate. Analyze the fractal character of asphalt mixture aggregate. For dense-graded aggregate, the distribution of particles has one fractal model. But for gap-graded aggregate, the distribution of particles has two different multi-fractal models. Adopt $D_{\mathrm{c}}$ (fractal dimension of coarse aggregate) and $D_{\mathrm{f}}$ (fractal dimension of fine aggregate) as the indexes to describe the fractal characters of asphalt mixture aggregate. Fractal gradation theory is developed to calculate the amount of material in the system finer than size "r". This theory can be used for asphalt mixture aggregate gradation design not only for dense-graded aggregate, but also for gap-graded aggregate. Analyze the relationship between the fractal gradation theory and other gradation theories. The results showed that the fractal gradation theory can contain the others because fractal is the essence of aggregate.

Fractal theory is an interdisciplinary subject which can describe the characteristics of complex structure and space filling ability ${ }^{[1]}$. Fractal theory has the potential to provide us with a new way to understand and analyze natural spatial phenomena, which are not smooth, but rough and fragmented to self-similarity over a rang of scales ${ }^{[2]}$. Asphalt mixture is one kind of composite material with complex microstructure. Aggregates have typical self-similarity. This paper studied the gradation theory of asphalt mixture aggregates by using the fractal theory.
\end{abstract}

\section{The calculation method of fractal dimension of asphalt mixture aggregate}

In 2007, YANG Rui-hua and XU Zhi-hong ${ }^{[3]}$ introduce an approach to calculating fractal dimension of dense-graded aggregate:

$$
P(r)=\frac{r_{\min }^{3-D}-r^{3-D}}{r_{\min }^{3-D}-r_{\max }^{3-D}}
$$

Percentage passing " $\mathrm{r}$ ” can be got by using formula (1). Percentage passing maximum particle size is always $100 \%$ and Percentage passing nominal maximum particle size is usually in the range of $90 \% \sim 100 \%$. In order to control the percentage passing nominal maximum particle size sieve, in formula (1), $r_{\max }$ is replaced by NMPS (normal maximum particle size). So the fractal model of grain size distribution can be represented in the form:

$$
P(r)=\frac{r_{\min }^{3-D}-r^{3-D}}{r_{\min }^{3-D}-N M P S^{3-D}} P_{0}
$$

Where $\mathrm{P}_{0}$ is the percentage passing NMPS and is always in the range of $90 \% \sim 100 \%$.

The slop of $\lg P(r)$ versus $\lg (r / \mathrm{NMPS})$ graph is 3-D. 


\section{The fractal characters of asphalt mixture aggregate}

\subsection{The fractal dimension of asphalt mixture aggregate}

Fractal dimension is the key parameter which can describe fractal character. By using fractal dimension and correlated coefficients $\mathrm{R}^{2}$, the regularity of aggregate gradation can be disclosed. The $\lg P(r)$ versus $\lg (r /$ NMPS) graphs of 12 gradations which are PR Chinese specification upper and lower limits for DAC (dense asphalt mixture) and SMA ${ }^{[4]}$.are shown in fig (1):

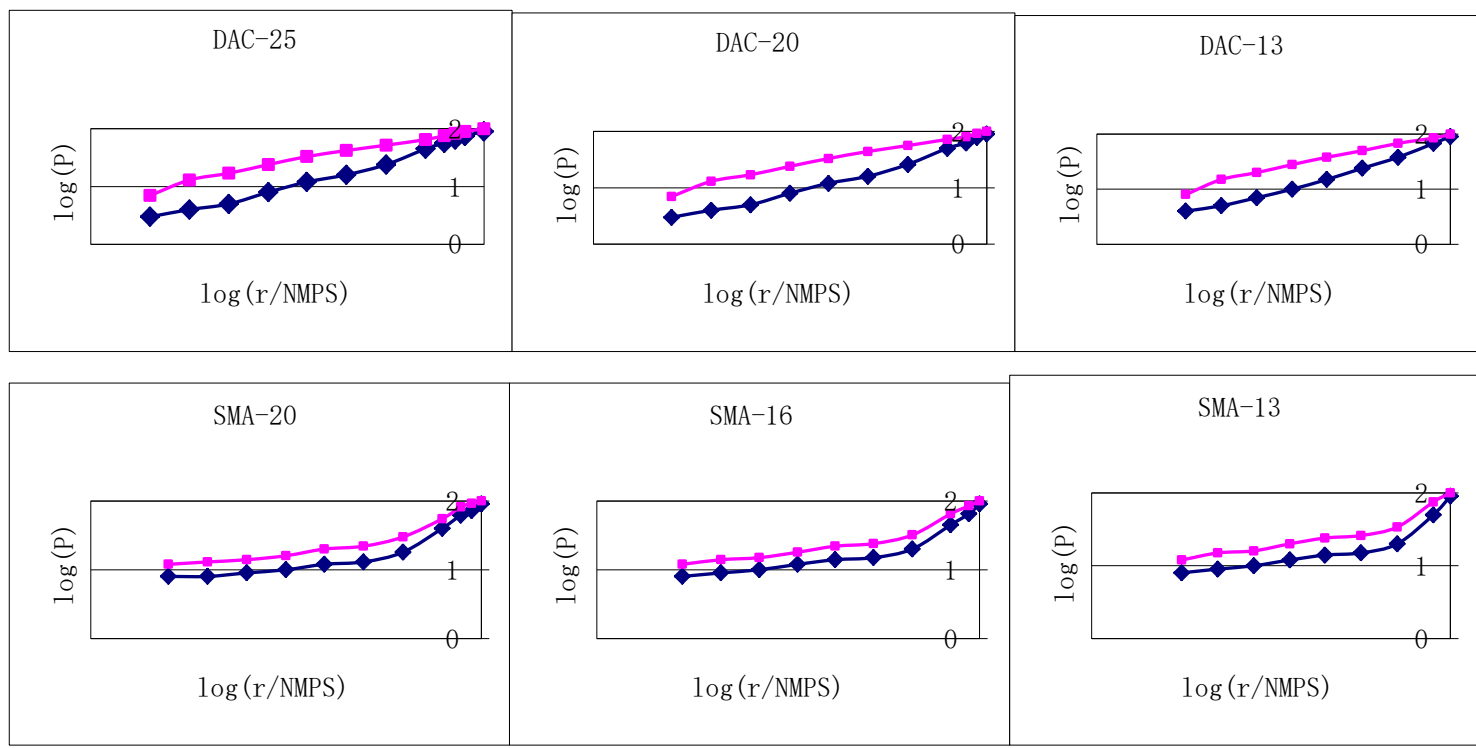

- lower limit

- upper limit

Fig.1 $\lg \mathrm{P}(\mathrm{r})$ versus $\lg (\mathrm{r} / \mathrm{NMPS})$ graphs of 12 gradations

The fractal dimensions of 12 gradations are listed in tab (1)

Table $1 \mathrm{D}$ of aggregate with 12 gradations

\begin{tabular}{cccc}
\hline gradation & Slop K & $D$ & $\mathrm{R}^{2}$ \\
\hline DAC-25upper limit & 0.4183 & 2.5817 & 0.985 \\
DAC-25 lower limit & 0.5987 & 2.4013 & 0.9896 \\
DAC-20 upper limit & 0.4428 & 2.5572 & 0.9877 \\
DAC-20 lower limit & 0.6238 & 2.3762 & 0.9839 \\
DAC-13 upper limit & 0.459 & 2.541 & 0.9854 \\
DAC-13 lower limit & 0.611 & 2.389 & 0.9858 \\
SMA-20 upper limit & 0.3963 & 2.6037 & 0.8914 \\
SMA-20 lower limit & 0.4397 & 2.5603 & 0.8445 \\
SMA-16 upper limit & 0.3835 & 2.6165 & 0.8945 \\
SMA-16 lower limit & 0.416 & 2.584 & 0.8485 \\
SMA-13 upper limit & 0.372 & 2.628 & 0.8843 \\
SMA-13 lower limit & 0.4024 & 2.5976 & 0.8052 \\
\hline
\end{tabular}

In rang of $r_{\min }$ to NMPS, the fractal dimension of upper limit gradation is higher than the fractal dimension of the lower limit gradation. The correlated coefficients $\mathrm{R}^{2}$ of DAC are higher 
than SMA. The quantity of this coefficient reflects the fitting degree for fractal ideal model. For SMA, the intermediate size of the overall aggregate gradation was removed and it broke the ideal fractal structure into several fractal structures. Analysis the $\lg P(r)$ versus $\lg (r /$ NMPS) graph of SMA and it can be seen that $4.75 \mathrm{~mm}$ sieve is the point of discontinuity in the regression curve. $4.75 \mathrm{~mm}$ is the sieve of dividing coarse and fine. So for gap-graded aggregate, it is more reasonable to describe the fractal characters in two ranges such as $4.75 \mathrm{~mm} \sim \mathrm{NMPS}$ and $\mathrm{r}_{\min } \sim 4.75 \mathrm{~mm}$. The $\mathrm{D}$ in the two different rang which were got by using subsection regression method were listed in tab (2).

Table 2 D of SMA aggregate

\begin{tabular}{|c|c|c|c|c|c|c|c|c|}
\hline \multirow[t]{2}{*}{ gradation } & \multicolumn{3}{|c|}{$\leq 4.75 \mathrm{~mm}$} & \multicolumn{3}{|c|}{$\geq 4.75 \mathrm{~mm}$} & \multirow{2}{*}{$\begin{array}{c}\text { average } \\
\text { D }\end{array}$} & \multirow{2}{*}{$\begin{array}{c}\text { average } \\
\mathrm{R}^{2}\end{array}$} \\
\hline & Slop K & $D$ & $\mathrm{R}^{2}$ & Slop K & $D$ & $\mathrm{R}^{2}$ & & \\
\hline $\begin{array}{l}\text { SMA-20 } \\
\text { upper limit }\end{array}$ & 0.2151 & 2.7849 & 0.9524 & 0.9029 & 2.0971 & 0.9901 & 2.4410 & 0.97125 \\
\hline $\begin{array}{l}\text { SMA-20 } \\
\text { lower limit }\end{array}$ & 0.1910 & 2.8090 & 0.9267 & 1.1616 & 1.8384 & 0.9981 & 2.3237 & 0.9624 \\
\hline $\begin{array}{c}\text { SMA-16 } \\
\text { upper limit }\end{array}$ & 0.2279 & 2.7721 & 0.9786 & 0.9416 & 2.0584 & 0.997 & 2.41525 & 0.9878 \\
\hline $\begin{array}{c}\text { SMA-16 } \\
\text { lower limit }\end{array}$ & 0.2125 & 2.7875 & 0.98 & 1.2111 & 1.7889 & 0.9963 & 2.2882 & 0.98815 \\
\hline $\begin{array}{l}\text { SMA-13 } \\
\text { upper limit }\end{array}$ & 0.2396 & 2.7604 & 0.9856 & 1.0688 & 1.9312 & 0.9964 & 2.3458 & 0.991 \\
\hline $\begin{array}{l}\text { SMA-13 } \\
\text { lower limit }\end{array}$ & 0.2125 & 2.7875 & 0.98 & 1.4485 & 1.5515 & 0.994 & 2.1695 & 0.987 \\
\hline
\end{tabular}

For SMA, D of aggregate in rang of 0.075 NMPS is higher than average D in the two different rang. This proved that the particles of SMA is bigger than of DAC in the same rang of 0.075 NMPS. Correlated coefficients $\mathrm{R}^{2}$ of SMA in tab (2) is higher than $\mathrm{R}^{2}$ in tab (1). This proved that it is reasonable to describe the fractal characteristics in two ranges.

\subsection{Fractal character of asphalt mixture aggregate}

For dense-graded aggregate, the distribution of particles can be described in one fractal dimension. But for gap-graded aggregate, the distribution of particles must be describe in $D_{\text {c }}$ (fractal dimension of coarse aggregate) and $D_{\mathrm{f}}$ (fractal dimension of fine aggregate) The fractal character of dense-graded aggregate and gap-graded aggregate are shown in fig (2). 


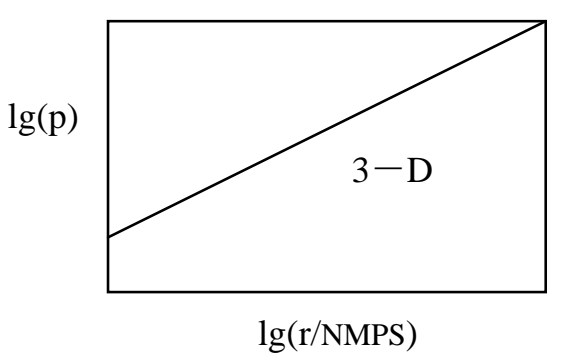

Fractal character of dense-graded aggregate

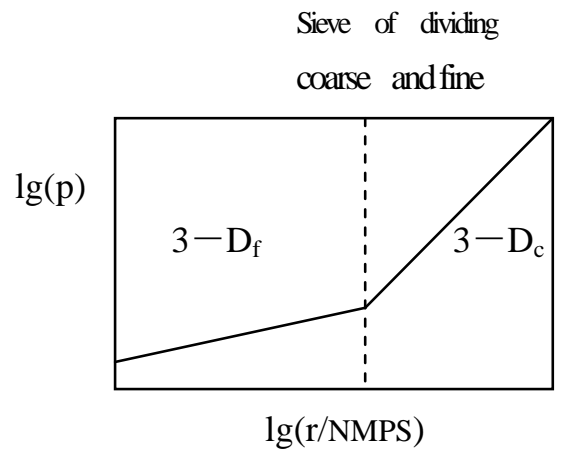

Fractal character of gap-graded aggregate

Fig.2 Fractal character of aggregate

In order to unify the fractal models for dense-graded aggregate and gap-graded aggregate, $D_{\mathrm{c}}$ and $D_{\mathrm{f}}$ are adopted to describe the fractal character not only for dense-graded aggregate but also for gap-graded aggregate.

\section{Fractal gradation theory}

For any kind of asphalt mixture aggregate with fractal character, the fractal dimension $\mathrm{D}$ will be got if we know $\mathrm{P}(\mathrm{r})$ which is percentage passing "r". Conversely, $\mathrm{P}(\mathrm{r})$ can be got by using the fractal gradation model..

By using formula (2), P(r) for dense-graded aggregate can be easily got. But for gap-graded aggregate, which has two fractal dimensions, $\mathrm{P}(\mathrm{r})$ can't be calculated directly by using formula (2). For the coarse aggregate of gap-graded aggregate, in the rang of DCF (sieve of dividing coarse and fine) to NMPS, $r_{\min }=D C F$. But for fine aggregate of gap-graded gradation, in the rang of $r_{\min }$ to DCF (sieve of dividing coarse and fine), NMPS $=\mathrm{DCF}$ and $\mathrm{P}_{0}=\mathrm{P}(\mathrm{DCF})$.. So the fractal gradation model for gap-graded aggregate can be represented in the formula (3):

$$
\left\{\begin{array}{c}
P=\frac{r_{\min }^{3-D_{C}}-r^{3-D_{C}}}{r_{\min }^{3-D_{C}}-N M P S^{3-D_{C}}} P_{0} \quad r \in(D C F, N M P S) \\
P=\frac{r_{\min }^{3-D_{f}}-r^{3-D_{f}}}{r_{\min }^{3-D_{f}}-N M P S^{3-D_{f}}} \times \frac{r_{\min }^{3-D_{C}}-D C F^{3-D_{C}}}{r_{\min }^{3-D_{C}}-N M P S^{3-D_{C}}} \times \frac{r_{\min }^{3-D_{f}}-D C F^{3-D_{f}}}{r_{\min }^{3-D_{f}}-N M P S^{3-D_{C}}} P_{0} \quad r \in\left(r_{\min }, D C F\right)
\end{array}\right.
$$

Because $r_{\min }^{3-D} \Rightarrow 0$, so in order to simplify the formula (3), delete $r_{\min }^{3-D}$ in the empirical equations and the reduced fractal model for gap-graded aggregate is:

$$
\begin{cases}P=\left(\frac{r}{N M P S}\right)^{3-D_{C}} \cdot P_{0} & r \in(D C F, N M P S) \\ P=\left(\frac{r}{D C F}\right)^{3-D_{f}} \cdot\left(\frac{D C F}{N M P S}\right)^{3-D_{C}} \cdot P_{0} & r \in\left(r_{\min }, D C F\right)\end{cases}
$$


Where:

$D C F=$ sieve of dividing coarse and fine

NMPS = normal maximum particle size

$D_{C}=$ fractal dimension in the rang of $D C F \sim N M P S$

$\mathrm{D}_{\mathrm{f}}=$ fractal dimension in the rang of $r_{\min } \sim D C F$

When $D_{f}=D_{C}=D$, the $\mathrm{P}(\mathrm{r})$ calculated by fig(4) is consistent with $\mathrm{P}(\mathrm{r})$ calculated by fig(2). So fig (4) is the general formula of fractal model not only for dense-graded aggregate but also for gap-graded aggregate.

\section{Relationships between the fractal gradation theory and other gradation theory}

\subsection{Maximum density gradation theory}

Fuller did the groundbreaking work on adjusting gradation to render the greatest strength and workability. He developed an ideal shape of the gradation curve and the idea that aggregate gradation could be controlled and thus affect concrete properties led to other research and ultimately to specifications governing aggregate gradation. Work by Wig, et al. suggested that Fuller's conclusions could not necessarily be extrapolated to aggregates different from the ones used in the original study. It was shown that the Fuller curve may not always give the maximum strength or maximum density (Wig et al. 1916). Talbot developed the well known equation ${ }^{[5]}$ :

$$
P=\left[\frac{r}{r_{\max }}\right]^{n}
$$

Where:

$r=$ size of the particular group in question

$\mathrm{r}_{\max }=$ largest particle in the system

$\mathrm{n}=$ exponent governing the distribution of sizes.

Their work indicated that for a given maximum particle size $r_{\max }$, the equation produces the maximum density when $\mathrm{n}=0.5$.

Taking the logarithm of each side of the equation, equation (6) was obtained:

$$
\log (P)=n \times \log \left(\frac{r}{r_{\max }}\right) .
$$

Because $3-D=\lambda=\mathrm{n}$, so $D=3$-n. It can be seen clearly that the equation (5) which was developed by Talbot is the special case of equation (4) when $D_{\mathrm{f}}=D_{C}=D$.

\subsection{Lin Xiu-xian gradation theory}

Lin Xiu-xian developed the gradation theory which put forward passing percentage decreasing coefficient $\mathrm{i}$ as main parameter in the gradation calculation formula ${ }^{[6]}$ :

$$
P=100 i^{x}
$$

Where:

$\mathrm{i}=$ passing percentage decreasing coefficient

$\mathrm{X}=$ series of particular size $\mathrm{X}=3.32 \lg \left(r_{\max } / r_{\mathrm{x}}\right)$

Taking the logarithm of each side of the equation, equation (8) was obtained: 


$$
\log (P)=x \times \log (i)+2=3.32 \log \left(r_{\max } / r_{x}\right) \times \log (i)+2=-3.32 \log \left(r_{x} / r_{\max }\right) \times \log (i)+2
$$

We can obtain $3-\mathrm{D}=-3.32 \log (i)$. It can be seen that the equation (6) is another form of equation (4).

\subsection{Ivanov gradation theory}

Ivanov developed the gradation theory which put forward mass decreasing coefficient $\mathrm{k}$ as main parameter in gradation calculation formula. When adjacent granules decrease with a series of $1 / 2$, the gradation calculation formula is ${ }^{[6]}$ :

$$
P_{x}=100 \times\left[1-\frac{1-K^{x}}{1-K^{m}}\right]
$$

Where:

$x=$ series of particular size and $\mathrm{x}=3.32 \lg \left(r_{\max } / r_{\mathrm{x}}\right)$

$r_{x}=$ the size of the $\mathrm{x}$ particular

$\mathrm{k}=$ mass decreasing coefficient

$P_{x}=$ amount of material in the system finer than size series “ $x$ "

Taking the logarithm of each side of the equation, relational expression (10) was obtained.

$$
\log (P(r)) \propto 3.32 \log \left(\frac{r_{\max }}{r_{x}}\right) \log (K)
$$

We can obtain 3-D=-3.32log $(K)$. It can be seen clearly that the equation (7) is another form of formal (4) which takes into account not only the condition that adjacent granules decrease with a series of $1 / 2$ but also the other conditions.

\subsection{Gradation theory of particle interference}

Weymouth based his theory on the size of the space between particles and the next smaller size which would fit into the space. For maximum workability and the greatest economy, the relationship between particle sizes should be one in which particles of one size group ate just under the opening provide by the next larger particle group ${ }^{7}$. In the reference ${ }^{[8]}$, gradation calculation formula for dense-graded aggregate was concluded by using Weymouth's theory of particle interference as follow:

$$
P=100 \times\left(1-\rho_{a}\right)^{x}
$$

Where:

$\mathrm{X}=$ series of particular size , and $\mathrm{x}=3.32 \lg \left(r_{\max } / r_{\mathrm{x}}\right)$ when adjacent granules decrease with a series of $1 / 2$.

$\rho_{\mathrm{a}}=$ ratio of the absolute volume of a size group to the space available to that size in the aggregate.

$$
P=100 \times\left(1-\rho_{a}\right)^{x} \Rightarrow \log (P)=x \times \log \left(1-\rho_{a}\right)+2 \Rightarrow 3-D=-3.32 \log \left(1-\rho_{a}\right)
$$

It can be seen that the gradation formula of particle interference has essential connections with fractal gradation theory.

\subsection{Bailey gradation method}

The Bailey method was originally developed by Robert D. Bailey of the Illinois Department of Transportation in the early 1980s. It is a practical tool that has been successfully utilized for developing and analyzing hot asphalt mixes in the lab and field ${ }^{[9]}$.

Four sieves are evaluated under the Bailey Method: the half sieve (NMPS/2), the primary control sieve (PCS), the secondary control sieve (SCS), and the tertiary control sieve (TCS). 
PCS $=0.22$ NMPS, SCS $=0.22$ PCS, and TCS $=0.22$ SCS.

The Bailey Method uses three ratios of the various sieves above to control the final gradation. The ratios are as follows:

$$
\begin{aligned}
& \mathrm{CA}=\left(P_{N M P S / 2}-P_{P C S}\right) /\left(100-P_{N M P S / 2}\right) \\
& F A C=\frac{P_{F 1}}{P_{P C S}} \\
& F A F=\frac{P_{F 2}}{P_{F 1}}
\end{aligned}
$$

Where

CA is the coarse aggregate ratio; FAC is the fine aggregate coarse ratio; and FAF is the fine aggregate fine ratio. $\mathrm{P}_{\mathrm{NMPS} / 2}$ is the percentage passing half sieve. $\mathrm{P}_{\mathrm{PCS}}$ is the percentage passing PCS. $\mathrm{P}_{\mathrm{F} 1}$ is the percentage passing SCS and $\mathrm{P}_{\mathrm{F} 2}$ is the percentage passing TCS.

Analyze the three ratios by using the fractal gradation theory. Study the relationship between the three ratios and fractal dimension.

Bailey Method use PCS to differentiate between the coarse and fine aggregate and

DCF=PCS $=0.22 \mathrm{NMPS}$, so $\mathrm{P}_{\mathrm{PCS}}$ and $\mathrm{P}_{\mathrm{NMPS} / 2}$ can be presented as follow by using fractal gradation theory:

$$
\begin{aligned}
& P_{(P C S)}=\left(\frac{P C S}{N M P S}\right)^{3-D_{C}} \cdot P_{0}=(0.22)^{3-D_{C}} \cdot P_{0} \\
& P_{(N M P S / 2)}=\left(\frac{N M P S / 2}{N M P S}\right)^{3-D_{C}} \cdot P_{0}=(0.5)^{3-D_{C}} \cdot P_{0}
\end{aligned}
$$

So the CA is:

$$
C A=\left(P_{N M P S / 2}-P_{P C S}\right) /\left(100-P_{N M P S / 2}\right)=\frac{0.5^{3-D_{C}} \cdot P_{0}-0.22^{3-D_{C}} \cdot P_{0}}{100-0.5^{3-D_{C}} \cdot P_{0}}
$$

It can be seen clearly that CA is the function of $D_{C}$, and $P_{0}$ which is $\%$ passing NMPS.

On the same way, $P_{F 1}$ and $P_{\mathrm{F} 2}$ can be presented as follows:

$$
\begin{aligned}
& P_{F 1}=(0.22)^{6-D_{C}-D_{f}} \cdot P_{0} \\
& P_{F 2}=(0.22 \times 0.22)^{3-D_{f}} \cdot(0.22)^{3-D_{C}} \cdot P_{0}
\end{aligned}
$$

So,

$$
\begin{gathered}
F A C=\frac{P_{F 1}}{P_{P C S}}=(0.22)^{3-D_{f}} \\
F A F=\frac{P_{F 2}}{P_{F_{1}}}=(0.22)^{3-D_{f}}
\end{gathered}
$$

FAC and FAF are the functions of $\mathrm{D}_{\mathrm{f}}$

From the above analysis, we can see that the three ratios (CA , FAC and FAF) are the function of fractal dimensions. So the three ratios which is pointed up by Bailey method and fractal dimension are the same essentially.

\subsection{Summary of gradation theories}

The relationship between the fractal gradation theory and other gradation theories are shown in tab (3): 
Table 3 The relationship between fractal gradation theory and other gradation theories

\begin{tabular}{|c|c|c|}
\hline gradation theory & Key parameters & $\begin{array}{l}\text { relationship between key } \\
\text { parameters and fractal } \\
\text { dimension }\end{array}$ \\
\hline Maximum density theory & $\mathrm{n}$ & $D=3-n$ \\
\hline $\begin{array}{c}\text { Lin Xiu-xian gradation } \\
\text { theory }\end{array}$ & $\mathrm{i}$ & $3.32 \lg (\mathrm{i})=\mathrm{D}-3$ \\
\hline Ivanov gradation theory & $\mathrm{K}$ & $3.32 \lg (\mathrm{K})=\mathrm{D}-3$ \\
\hline particle interference theory & $\rho_{\mathrm{a}}$ & $3.32 \lg \left(1-\rho_{a}\right)=D-3$ \\
\hline Bailey method & $\begin{array}{l}\text { CA } \\
\text { FAC } \\
\text { FAF }\end{array}$ & $\begin{array}{l}C A=\frac{0.5^{3-D_{C}} \cdot P_{0}-0.22^{3-D_{C}} \cdot P_{0}}{100-0.5^{3-D_{C}} \cdot P_{0}} \\
F A C=\frac{P_{F 1}}{P_{P C S}}=(0.22)^{3-D_{f}} \\
F A F=\frac{P_{F 2}}{P_{F_{1}}}=(0.22)^{3-D_{f}}\end{array}$ \\
\hline
\end{tabular}

Tab (3) showed that the fractal gradation theory can contain the others. The reason is mainly that: fractal is the essence of aggregate.

\section{Conclusion}

1. Analyze the fractal character of dense-graded aggregate and gap-graded aggregate. For dense-graded aggregate, the distribution of particles has one fractal model. But for gap-graded aggregate, the distribution of particles has two different multi-fractal models. In order to unify the fractal models of dense-graded aggregate and gap-graded aggregate, $\mathrm{D}_{\mathrm{c}}$ and $\mathrm{D}_{\mathrm{f}}$ are adopted to describe the fractal character whether for dense-graded aggregate or for gap-graded aggregate.

2. Fractal gradation method was put forward. Fig (4).can be used not only for designing of dense-graded aggregate, but also for gap-graded aggregate

3. Analyze the relationship between the fractal gradation theory and other gradation theories. The results showed that the fractal gradation method can contain the others. The reason is mainly that: fractal is the essence of aggregate.

\section{Reference:}

[1] LONG Qi-Yi , LONG Qi-Wei, MU Zai-Le , Idealized Fractal Model and Practical Nested Structure [J] Physics, 1994,23(3): 158 164 (in Chinese)

[2] TANG Ming; BA Heng-jing Evaluation of topologic and fractal characteristics of concrete [J] Journal of Harbin University of Civil Engineering and Architecture, 2002, 35 (1): 86-89 (in Chinese)

[3] YANG Rui-hua, XU Zhi-hong, Relationship between fractal dimension and road per formance of dense-gradation asphalt mixture [J] China Civil Engineering Journal 2007, 3: 98 109 (in Chinese)

[4] Ministry of Communications of PR China, Technical Specifications for Construction of 
Highway Asphalt Pavements JTG F40-2004 [s] Beijing: The People's Communication Press, 2004 (in Chinese))

[5] LI L H,ZHANG N L .Road Constructional Materials[M] Beijing: The People's Communication Press, 2004 (in Chinese)

[6] LIN-Xiuxian. Design Method of Flexible Road Surface Structure[M]. Beijing: People Traffic Press,1988 (in Chinese)

[7] Weymouth, C.A.G (1933) "Effect of Particle Interference in Mortars and Concrete.” Rock Products 36(2):26-30

[8] Yan Jia-Ji, Road constructional materials[M].Beijing:People's Communications Press 1986 (in Chinese)

[9] William R V, William J P, Samuel H C Bailey method for gradation selection in HMA mixture design [R]. Transportation Research Circular Number E-C044,2002 